\title{
Deep multicolor CCD photometry of the globular cluster NGC 6723 ${ }^{\star}$
}

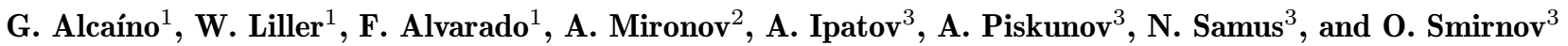 \\ ${ }^{1}$ Isaac Newton Institute, Ministry of Education of Chile, Casilla 8-9, Correo 9, Santiago, Chile \\ e-mail: inewton@reuna.cl \\ 2 Sternberg Astronomical Institute and Isaac Newton Institute Moscow Branch, 13 University Ave., Moscow 119899, Russia \\ e-mail: almir@sai.msu.su \\ ${ }^{3}$ Institute of Astronomy of Russian Acad. Sci. and Isaac Newton Institute Moscow Branch, 48 Pyatnitskaya Str., \\ Moscow 109017, Russia \\ e-mail: ipatov@inasan.rssi.ru, piskunov@inasan.rssi.ru, samus@sai.msu.su, oms@inasan.rssi.ru
}

Received November 17, 1998; accepted March 23, 1999

\begin{abstract}
We have obtained CCD $U B V R I$ photometry of 3620 stars in the globular cluster NGC 6723 , one of the few globular clusters with a horizontal branch wellpopulated on both sides of the instability strip. This is the first deep five-color study of NGC 6723. We have determined several important parameters for this cluster. Its color excess is $E(B-V)=0.11 \pm 0.01$. The most plausible value of metallicity is $[\mathrm{Fe} / \mathrm{H}]=-1.22 \pm 0.15$; even lower values are possible. The apparent distance modulus of the cluster is $(m-M)_{V, 6723}=14.76$. From isochrone analysis, we prefer the NGC 6723 age in the range between 15 and 16 Gyr, in the scale of Bergbusch \& VandenBerg (1992). We find a close similarity of the shape and slope of the red giant branch in NGC 6723 and M 3 and of the magnitude difference between the main-sequence turnoff and the horizontal branch in NGC 6723, M 3, and M 5.
\end{abstract}

Key words: globular clusters: individual (NGC 6723)

\section{Introduction}

Color-magnitude diagrams (CMDs) of globular clusters (GCs) can be subdivided into three main groups according to the appearance of their horizontal branches (HBs). The morphology of a HB can be numerically expressed using either the parameter $B /(B+R)$ (Rosino 1965; Mironov $1972)$ or the parameter $R_{\mathrm{HB}}=(B-R) /(B+R R+R)$

Send offprint requests to: G. Alcaíno

* Based on observations collected at the Las Campanas Observatory, Carnegie Institution of Washington.

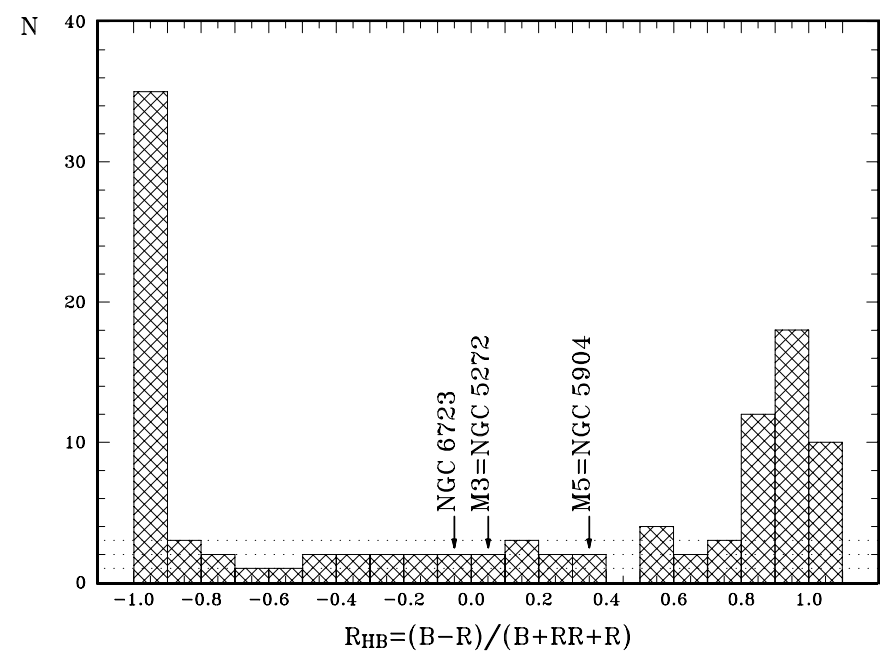

Fig. 1. The distribution of the globular clusters of our Galaxy over the morphological parameter of the horizontal branch, $R_{\mathrm{HB}}$

(Lee et al. 1988), where $B$ and $R$ are respectively numbers of HB stars to the blue and to the red of the instability strip and $R R$ is the number of RR Lyrae variables. Figure 1 shows the distribution of the GCs of our Galaxy over $R_{\mathrm{HB}}$; the parameter values are from the catalog by Harris (1996).

The first group consists of clusters with "blue" HBs, with all HB stars to the blue of the RR Lyrae instability strip. The values of $B /(B+R)$ and $R_{\mathrm{HB}}$ for these clusters are close to 1 . These are, as a rule, very metal-poor GCs of the galactic halo, with typically $[\mathrm{Fe} / \mathrm{H}]=-1.5$ to -2.1 .

The second group consists of clusters with "red" HBs, with all $\mathrm{HB}$ stars to the red of the RR Lyrae instability 
strip. For such clusters, $B /(B+R)=0$, and $R_{\mathrm{HB}}=-1$. Usually these are clusters of the Galaxy's disk or central region, with typically $[\mathrm{Fe} / \mathrm{H}]=-0.5$ to -1.0 .

Finally, the third group contains GCs with HBs populated on both sides of the instability strip. This group is very heterogeneous both by HB morphology as well as by metallicity. Studying these clusters may give us a key to the "second parameter" problem.

The cluster studied in this paper, NGC $6723\left(18^{\mathrm{h}} 599^{\mathrm{m}} 6\right.$, $\left.-36^{\circ} 38^{\prime}, 2000.0\right)$, is one of the clusters of the third group. It is a southern globular cluster located towards the galactic center, at about $2.4 \mathrm{kpc}$ distance from the galactic plane, in a field with low interstellar reddening: the cata$\log$ of Harris (1996) gives $E_{B-V}=0.05$.

The first photographic photometry of stars in NGC 6723 was published by Gascoine \& Ogston (1963). More thorough studies were later undertaken by Menzies (1974) and Martins \& Fraquelli (1987). The first estimates of the cluster's integrated spectral type (Kinman 1959; Kron \& Mayall 1960) were G2-G4. Smith (1981), from the spectroscopic metal-abundance parameter $\Delta S$ derived for five RR Lyrae stars, found $\Delta S=2.8$ for NGC 6723, corresponding to $[\mathrm{Fe} / \mathrm{H}]=-0.68$ in the metallicity scale of Butler (1975). However, later studies showed a more significant metal deficiency. Smith \& Hesser (1986), from DDO photometry, determined $[\mathrm{Fe} / \mathrm{H}]=-1.1$. A similar value, $[\mathrm{Fe} / \mathrm{H}]=-1.1$, was found by Zinn \& West (1984) and Lee (1992). Recently Fullton and Carney (Fullton \& Carney 1993; Fullton \& Carney 1996; Fullton 1996) obtained 2-color $(B, V)$ CCD photometry of almost 18000 NGC 6723 stars, down to the 24th magnitude; the results of this study have not yet been published in the complete form. Fullton and Carney also obtained spectra $(\lambda \lambda 5000-7800 \AA)$ of three red giants in NGC 6723 , calibrated them with model atmospheres of Kurucz (1993), CD-ROM and, as a result, obtained still lower metal abundance, $[\mathrm{Fe} / \mathrm{H}]=-1.26$. Harris $(1996)$ catalogs $[\mathrm{Fe} / \mathrm{H}]=$ -1.12 (in the Zinn \& West 1984, scale), along with F9 for the cluster's integrated spectral type.

The present study is the first deep five-color study of NGC 6723. It enabled us to determine a number of important parameters for this cluster.

\section{Observations and reductions}

The observations reported on here were acquired on two nights, June 13/14 and 15/16, 1994, using the 100" telescope of the Las Campanas Observatory. The detector was a TEKTRONIC 2 CCD $(1024 \times 1024$ pixels $)$ with a readout noise of $8 \mathrm{e}^{-}$rms; the pixel size was $21 \mu \mathrm{m}$. The center of the measured field is approximately in $2^{\prime}$ to the east and $1^{\prime}$ to the north of the cluster center; a $4 \times 4$ arcmin field is covered by the frames. We used a total of 10 frames in $U$ (exposure time from $50^{\mathrm{s}}$ to $\left.900^{\mathrm{s}}\right), 12$ frames in $B\left(12^{\mathrm{s}}\right.$ to $\left.500^{\mathrm{s}}\right), 11$ frames in $V\left(10^{\mathrm{s}}\right.$ to $\left.360^{\mathrm{s}}\right), 12$ frames in $R\left(20^{\mathrm{s}}\right.$ to
Table 1. Observations of photoelectric standards in NGC 6723

\begin{tabular}{cccccc}
\hline & $V$ & $B-V$ & $U-B$ & $V-R$ & $V-I$ \\
\hline $\mathrm{C}$ & 12.926 & 1.612 & 1.858 & 0.847 & 1.645 \\
& -0.02 & +0.01 & -0.04 & -0.00 & +0.00 \\
$\mathrm{~F}$ & 13.257 & 1.478 & 1.648 & 0.772 & 1.513 \\
& -0.00 & -0.01 & -0.01 & -0.01 & -0.01 \\
$\mathrm{H}$ & 14.274 & 0.435 & -0.158 & 0.270 & 0.582 \\
& +0.01 & +0.00 & -0.10 & +0.00 & +0.01 \\
$\mathrm{~J}$ & 14.604 & 0.737 & 0.180 & 0.386 & 0.806 \\
& -0.04 & -0.00 & +0.02 & -0.03 & -0.00 \\
$\mathrm{~K}$ & 14.687 & 1.022 & 0.731 & 0.556 & 1.105 \\
& +0.05 & -0.02 & +0.03 & -0.01 & +0.01 \\
$\mathrm{M}$ & 15.463 & 0.983 & 0.521 & 0.561 & 1.112 \\
& +0.05 & +0.01 & +0.05 & +0.03 & +0.03 \\
$\mathrm{~N}$ & 15.374 & 0.687 & 0.032 & 0.411 & 0.868 \\
& -0.05 & -0.00 & -0.01 & +0.02 & -0.02 \\
$\mathrm{P}$ & 15.453 & 1.006 & 0.522 & 0.552 & 1.122 \\
& -0.01 & +0.01 & +0.06 & -0.02 & -0.03 \\
\hline & & & & &
\end{tabular}

$\left.420^{\mathrm{s}}\right)$, and 12 frames in $I\left(8^{\mathrm{s}}\right.$ to $\left.360^{\mathrm{s}}\right)$. The average seeing estimated during reductions was $1.5^{\prime \prime}$.

The reductions of the photometry were performed at the Institute of Astronomy (Moscow) using the software described by us earlier (Samus et al. 1995a) and based upon the DAOPHOT II ALLSTAR application (Stetson 1991). PSF stars, 15 to 20 per frame, bright but far from saturation, were selected interactively among those having no neighbors or defects within the PSF radius.

Our experience shows that, among standard PSFs provided by DAOPHOT, the PENNY2 function enables us to handle aberrations specific to individual frames most effectively. The photometric errors showed no significant dependence on the position within the frame, so we did not introduce any variations of the PSF.

In the reductions, we used photoelectric standards from Alvarado et al. (1994). As usual for CCD studies of the Isaac Newton Institute, we did not observe any comparison fields, but applied a photoelectric sequence established in the program field itself. The CCD magnitudes and colors of the eight used standards, which cover a rather wide range of color indices, are listed in Table 1, along with the deviations (in the sense CCD minus photoelectric) from the photoelectric values.

The formulae used in this study to derive magnitudes and color indices in the standard system from DAOPHOT ALLSTAR instrumental magnitudes for NGC 6723 are:

$$
\begin{aligned}
V & =v-0.015( \pm 0.040)(b-v)-0.017( \pm 0.041) \\
V & =v-0.015( \pm 0.038)(v-i)-0.015( \pm 0.046) \\
B-V & =1.063( \pm 0.012)(b-v)-0.032( \pm 0.013) \\
U-B & =1.081( \pm 0.032)(u-b)-0.008( \pm 0.028) \\
V-I & =0.922( \pm 0.025)(v-i)+0.048( \pm 0.025)
\end{aligned}
$$

In each photometric band, we retained only stars with at least two measurements. Consequently, we obtained magnitudes for 3620 stars in $V$ and $R, 3565$ stars in $B, 2307$ 

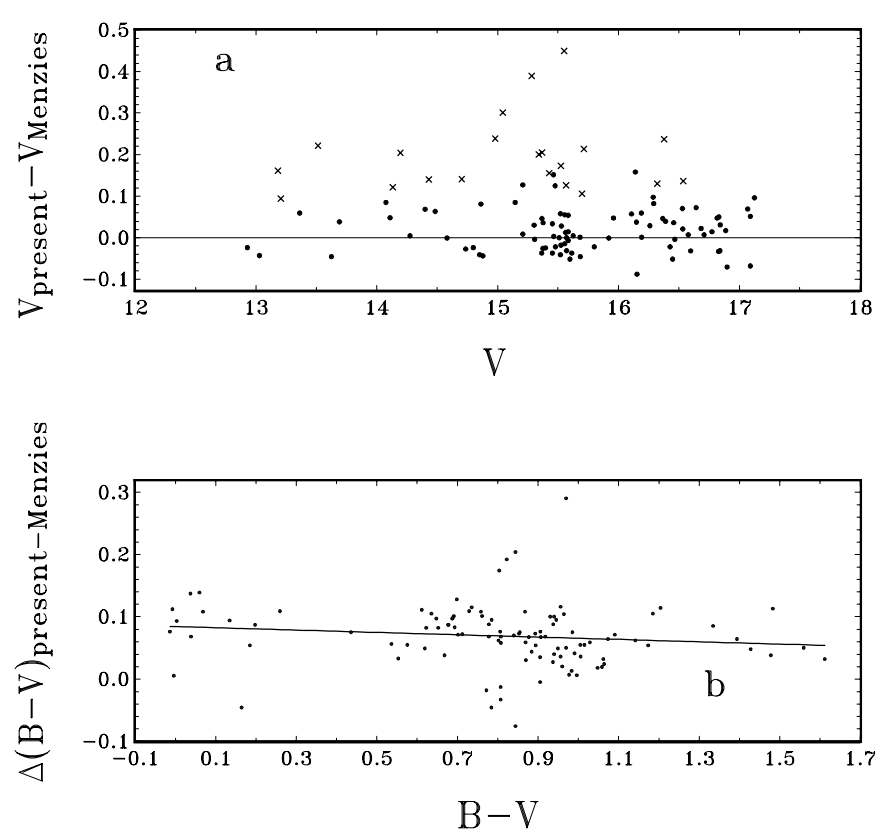

Fig. 2. Comparison of our photometry and that by Menzies (1974). a) Comparison of $V$ magnitudes; crosses, blends not separated by Menzies; b) comparison of $(B-V)$ colors. Our values are plotted along the $x$ axis; differences (in the sense our value minus that from Menzies) are plotted along $y$. The straight line in panel (2) is the regression $\Delta(B-V)=+0.084-$ $0.019(B-V)$

stars in $I$, and 2222 stars in $U$. The limiting $V$ magnitude was about 22 . The results of our photometry are available from N. Samus upon request.

For $V<15$, the random rms errors are, on average, $0^{\mathrm{m}} 021$ in $U ; 0 .{ }^{\mathrm{m}} 014$ in $B ; 0.013$ in $V ; 0.010$ in $R$; and 0 . 010 in $I$. For $15<V<18$, the errors are 0 . 030 in $U$; 0 . 021 in $B$; 0 . 016 in $V$; $0 .{ }^{\mathrm{m}} 011$ in $R$; and $0{ }^{\mathrm{m}} 014$ in $I$. For $V>18$, they are, on average, 0.088 in $U ; 0.055$ in $B$; $0{ }^{\mathrm{m}} 040$ in $V ; 0$. 033 in $R$; and 0.046 in $I$.

\section{Comparison with photographic photometry from Menzies 1974}

Figures 2a,b show the results of the comparison (based upon 108 stars in common) between our photometry and that published by Menzies (1974). It is clearly seen that, in some cases, the deviations of $V$ magnitudes are much larger than the errors of CCD and photographic photometry, reaching almost $0.5 \mathrm{mag}$, with magnitudes from Menzies always brighter. The deviations exceed 0.2 mag in almost 20 percent of the cases. Such deviations cannot be due to random errors. Actually, our analysis of individual deviant cases shows that they always occur when Menzies measured $a$ blend of two or more close stellar images, whereas our PSF photometry enables us to measure the magnitudes of the blended stars separately.

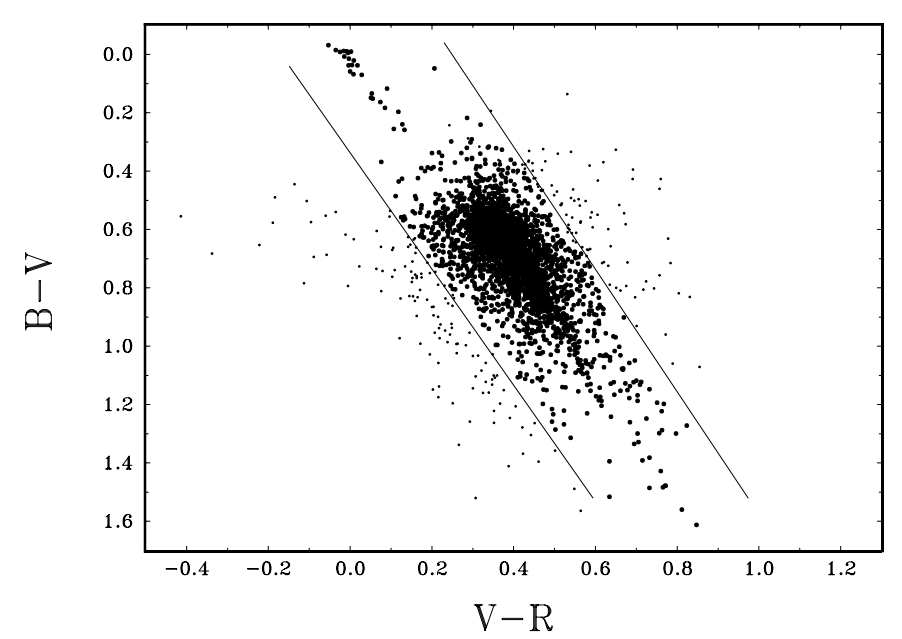

Fig. 3. The $(B-V, V-R)$ diagram for NGC 6723 (all stars). The straight lines and different symbols are explained in the text

If we reject all cases when Menzies reported combined magnitudes of blended photographic stellar images, we find that the standard deviation between the two sets of measurements is 0.05 , a typical random error of the photographic method. In most cases, we are able to reproduce combined magnitudes of blends measured by Menzies using our CCD magnitudes of their components, with deviations not exceeding 0.1 .

The comparison of $(B-V)$ color indices shows that the values given by Menzies are systematically bluer by approximately $00^{\mathrm{m}} 08$.

Unfortunately, magnitudes of the cluster's individual stars obtained in Martins \& Fraquelli (1987) and in Fullton \& Carney (1993), Fullton \& Carney (1996), Fullton (1996) have not been published. The CMDs shown in the figures in these papers are very similar to our results.

\section{Two-color diagrams. Metallicity and color excesses}

Multicolor photometry provides many possibilities for determinations of metallicity and reddening, two of the most important cluster parameters. $U B V R I$ photometry provides 10 different color indices, 4 of them independent, and hence 45 different two-color diagrams. Below some of them are discussed in detail.

\subsection{The $(B-V, V-R)$ diagram}

The $(B-V, V-R)$ diagram for NGC 6723 is shown in Fig. 3. It is clearly seen that numerous faint stars, with comparatively large photometric errors, deviate from the well-defined central line of the distribution. The group of stars lying parallel to the main strip, but below it and to 


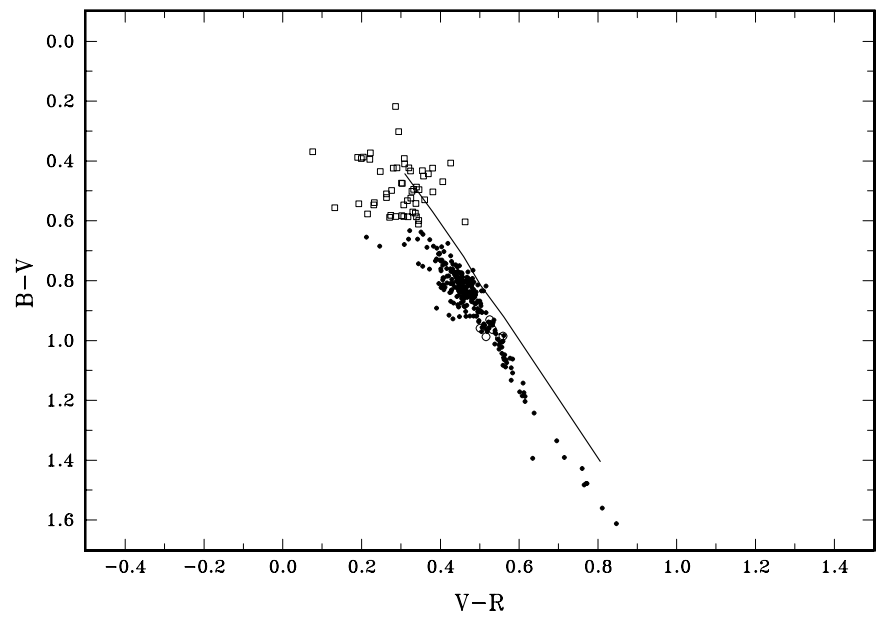

Fig. 4. The $(B-V, V-R)$ diagram for different sequences: stars of the RGB (filled circles), AGB (open circles), and the region of blue stragglers (open squares). The line is the theoretical giant sequence for $[\mathrm{Fe} / \mathrm{H}]=-1$

the left, is especially conspicuous. Such data points, with rather "blue" $(V-R)$ values, cannot correspond to any physical parameters of normal stars and supposedly are the result of photometric errors. All stars with this kind of error are located in the cluster's central part and, some of them, for reasons unclear to us, received, in our reductions, $V$ magnitudes that were too bright. Because of this, their $(B-V)$ values were found too "red" and $(V-R)$ values, too "blue". Similarly, data points significantly deviating upward and to the right of the main cloud correspond to the stars for which the derived $V$ magnitudes are too faint. Some of the data points to the right of the main cloud may belong to field stars; these stars do not show any noticeable concentration towards the cluster center. In the subsequent analysis, we did not take into account stars situated, in the $(B-V, V-R)$ plane, to the left or to the right of the area delimited in the figure by two parallel lines. Such stars have been plotted in Fig. 3 with smaller symbols.

Figure 4 presents the $(B-V, V-R)$ diagram for cluster stars of three sequences: the red-giant branch (RGB, filled circles), the asymptotic giant branch (AGB, open circles) and the region of blue stragglers (BSs, open squares). Most are among the cluster's brightest stars with best photometric accuracy. The straight line in the figure follows from computations of Buser \& Kurucz (1992) and corresponds to the giant sequence with $[\mathrm{Fe} / \mathrm{H}]=-1$. The vertical separation between the RGB and the theoretical sequence, at $(V-R)=+0.6$, is $\delta(B-V)=0.15$. According to calculations in Grebel \& Roberts (1995), based upon Kurucz's theoretical data, for moderately metal-deficient giants $([\mathrm{Fe} / \mathrm{H}]=-1.0)$ with $3500<T_{\text {eff }}<6000 \mathrm{~K}$ (i.e. in the range of color indices $\left.1.59>(B-V)_{0}>0.49\right)$, ratios of differential interstellar extinction are $E_{U-B} / E_{B-V}=$ $0.96 \pm 0.09, E_{V-R} / E_{B-V}=0.69 \pm 0.02, E_{R-I} / E_{B-V}=$

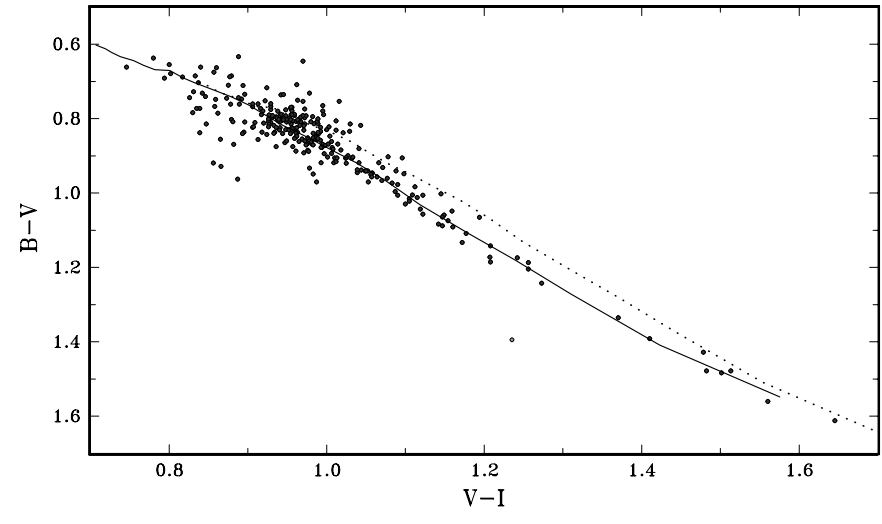

Fig. 5. The $(B-V, V-I)$ diagram for RGB stars in NGC 6723. Dotted line, the mean ridge line for M 3 according to Ferraro et al. (1996). Solid line, the same ridge line shifted as described in the text

$0.62 \pm 0.01, E_{V-I} / E_{B-V}=1.31 \pm 0.04$. Using the above value for $E_{V-R} / E_{B-V}$, and taking into account the theoretical slope of the giant branch $R_{B V, V R}=\mathrm{d}(V-$ $R) / \mathrm{d}(B-V)=0.516$, we find for NGC $6723 E_{B-V}=$ $0.11 \pm 0.01$ and $E_{V-R}=0.08 \pm 0.005$.

According to Grebel \& Roberts (1995), the decrease of metallicity from $[\mathrm{Fe} / \mathrm{H}]=0$ to $[\mathrm{Fe} / \mathrm{H}]=-2.0$ changes the slope of the giant branch in the $(B-V, V-R)$ diagram, $R_{B V, V R}$, from 0.534 to 0.512 . However, the observed value of this slope from our diagram is $\mathrm{d}(V-R) / \mathrm{d}(B-V) \approx$ 0.50 , somewhat outside the above range. This discrepancy does not significantly influence our determination of the differential interstellar extinction, but we cannot determine $[\mathrm{Fe} / \mathrm{H}]$ from such slope.

\subsection{The $(B-V, V-I)$ diagram}

Figure 5 shows the $(B-V, V-I)$ diagram for RGB stars in NGC 6723. Similar data were recently presented by Ferraro et al. (1996) for one of the best-studied clusters, M 3 = NGC 5272. The mean ridge lines from the cited paper make it possible to compare the giant branches of the two clusters in the $(B-V, V-I)$ plane. The dotted line in our diagram corresponds to M 3, and filled circles are our data. The $E_{B-V}$ color excess for M 3 is very low; according to different sources, it does not exceed 0. 03: Harris (1996) catalogs $E_{B-V}=0.01$, and Ferraro et al. (1996) adopt, for $\mathrm{M} 3, E_{B-V}=0$ m 00 . The solid line in Fig. 5 is the M 3 mean ridge line for the RGB, shifted by $\Delta_{B-V}=-0.10$ and $\Delta_{V-I}=-0$. 13 from its original position. This line apparently represents the data for NGC 6723 quite well; it repeats several bends of our diagram. Hence the NGC 6723 reddening relative to $\mathrm{M} 3$ is $\Delta E_{B-V}=0.10$ and $\Delta E_{V-I}=0.13$, and since $E_{B-V} \approx 0$ for $\mathrm{M} 3$, these values may be considered as color excesses for NGC 6723. The obtained values agree with those derived from the $(B-V, V-R)$ diagram as 


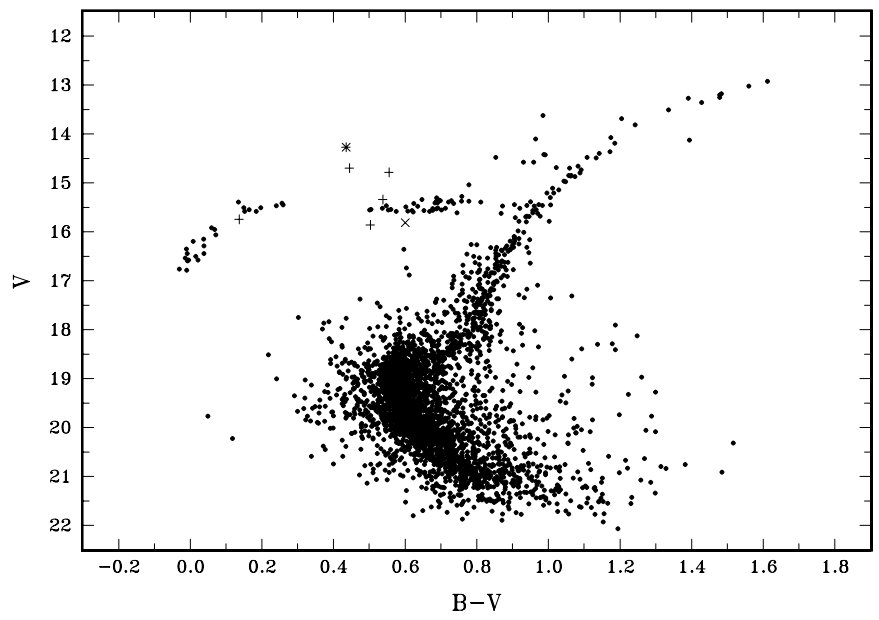

Fig. 6. The $(V, B-V)$ diagram for NGC 6723 . Plus signs, RR Lyrae variables, with their magnitudes measured at arbitrary phases; cross and asterisk, see explanation for Fig. 8

well as with the ratio of differential interstellar extinction $E_{V-I} / E_{B-V}=1.31$ calculated by Grebel \& Roberts (1995).

\section{Color-magnitude diagrams}

\subsection{Sequences in the $V, B-V$ diagram}

The $(V, B-V)$ diagram for NGC 6723 is presented in Fig. 6. All its sequences are well populated. Plus signs $(+)$ in Fig. 6 and in other diagrams designate five $R R$ Lyrae variables identified in our frames. Magnitudes of the variable stars in different bands were measured nonsimultaneously, so the plus signs in the diagram have arbitrary positions. The signs of two other types, the cross $(\times)$ and the asterisk, will be discussed below, when presenting Fig. 8. Table 2 summarizes $U B V R I$ ridge points of the principal sequences.

The cluster's horizontal branch in the $V, B-V$ diagram, in the color-index range $0.20<(B-V)<0.75$, actually runs almost exactly horizontally. The stars closest to the instability region and to the blue of it have, on average, $V=15.46$, slightly brighter than the stars immediately to the red of the instability region which have, on average, $V=15.50$. So we can accept $V_{\mathrm{HB}}=15.48$ as the magnitude of the horizontal branch at the level of the instability strip. This value coincides exactly with that given by Menzies (1974) and does not differ significantly from the value 15.4 published by Martins \& Fraquelli (1987).

Figure 7 illustrates the distribution of stars along the horizontal branch. This histogram shows that, at the HB level, the blue edge of the instability strip is at $(B-V)_{b}=$ $0.26 \pm 0.01$ and the red edge is at $(B-V)_{r}=0.50 \pm 0.01$. Menzies (1974) gives systematically bluer positions for the boundaries of the instability strip, namely $(B-V)_{b}=0.17$

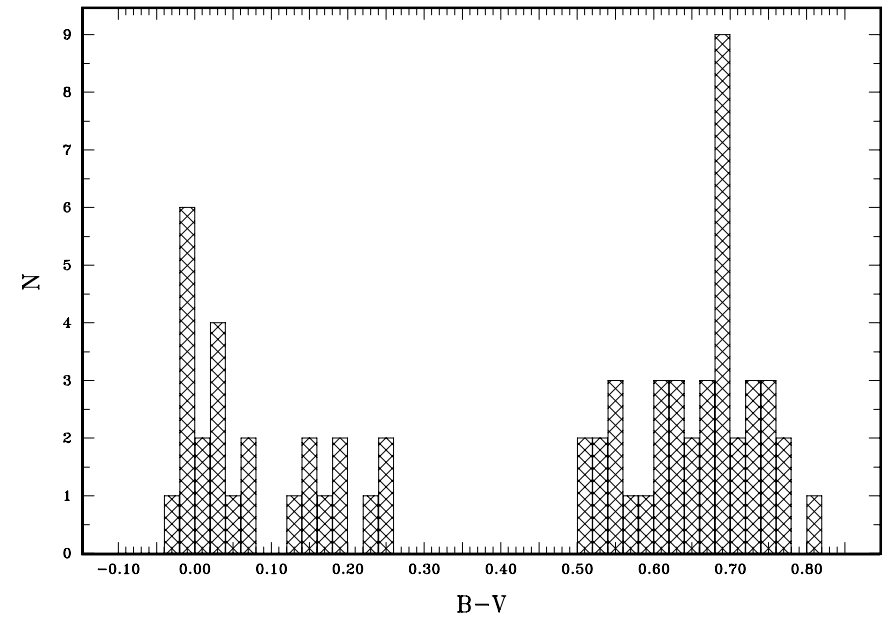

Fig. 7. The $(B-V)$ color distribution of stars along the HB of NGC 6723

and $(B-V)_{r}=0.44$, in agreement with the comparison of the photometries presented in Sect. 3 .

To derive magnitudes of the stars in NGC 6723 used in Martins \& Fraquelli (1987), "the photographic plates were first digitized using microdensitometer with a scanning aperture $40 \mu \mathrm{m}$ square" and then a PSF was derived and applied using the necessary software. However, Martins \& Fraquelli do not directly present their $(B-V)_{b}$ and $(B-V)_{r}$ values. From the distribution of stars along the horizontal branch of NGC 6723 published in their paper (Fig. 7), it follows that $(B-V)_{b} \approx 0.30$ and $(B-V)_{r} \approx 0.50$. However, these absolute values should be considered with caution. Figure $3 \mathrm{~b}$ in Martins \& Fraquelli (1987) shows that all their color indices are redder than in Menzies (1974), the difference at the blue edge of the instability strip being approximately 0.15 and at its red edge, 0.10 . Martins \& Fraquelli emphasize that their magnitudes of the photoelectric standards show good agreement with Menzies whereas the photographic magnitudes deviate considerably. We think that the discrepancy is the result of an insufficient account of systematic deviations between aperture and PSF photometry. Remarkably, within errors in our study and in studies of Menzies and of Martins \& Fraquelli, the measured widths of the instability strip are in good agreement.

The conspicuous maximum at $(B-V)_{1}=0.69 \pm 0.005$ apparent in Fig. 7 is noticeable as a clump of stars in the red horizontal branch in the diagrams published by Menzies and by Martins \& Fraquelli.

It has long been known (Mironov \& Samus 1974) that the dereddened color index of the most densely populated point on the horizontal branch, $(B-V)_{0,1}$, shows a good correlation with the difference of apparent (not dereddened) color indices of the same maximum and the blue edge of the instability strip, $\Delta_{b-1}=(B-V)_{1}-(B-V)_{b}$. This relation can be expressed as $(B-V)_{0,1}=0.154 \pm 0.008-(1.006 \pm 0.034) \Delta_{b-1}$, 
Table 2. Ridge lines of sequences in NGC 6723

\begin{tabular}{|c|c|c|c|c|c|c|c|c|c|}
\hline$V$ & $B-V$ & $V-R$ & $V-I$ & $U-B$ & $\bar{V}$ & $B-V$ & $V-R$ & $V-I$ & $U-B$ \\
\hline \multicolumn{5}{|c|}{$(\mathrm{MS}+\mathrm{SG}+\mathrm{RG})$} & \multicolumn{5}{|c|}{ HB } \\
\hline 20.90 & 0.798 & 0.467 & - & - & 16.73 & -0.017 & -0.028 & 0.048 & -0.615 \\
\hline 20.73 & 0.755 & 0.453 & - & - & 16.56 & -0.002 & -0.018 & 0.065 & -0.515 \\
\hline 20.60 & 0.731 & 0.440 & - & - & 16.40 & 0.026 & -0.012 & 0.078 & -0.433 \\
\hline 20.47 & 0.707 & 0.433 & - & - & 16.20 & 0.055 & -0.002 & 0.102 & -0.355 \\
\hline 20.34 & 0.682 & 0.423 & - & - & 15.95 & 0.079 & 0.023 & 0.150 & -0.240 \\
\hline 20.19 & 0.668 & 0.412 & - & - & 15.74 & 0.108 & 0.040 & 0.180 & -0.150 \\
\hline 20.00 & 0.649 & 0.385 & 0.660: & - & 15.64 & 0.137 & 0.060 & 0.240 & -0.100 \\
\hline 19.86 & 0.634 & 0.381 & 0.630: & -0.200 : & 15.56 & - & - & - & -0.080 \\
\hline 19.69 & 0.620 & 0.365 & 0.611: & $-0.205:$ & 15.55 & 0.166 & 0.075 & 0.277 & -0.075 \\
\hline 19.57 & 0.605 & 0.362 & 0.600: & -0.210 : & 15.52 & 0.547 & 0.300 & 0.575 & -0.060 \\
\hline 19.43 & 0.596 & 0.365 & 0.594: & -0.215 : & 15.52 & 0.591 & 0.320 & 0.600 & - \\
\hline 19.26 & 0.586 & 0.354 & 0.567 & -0.220 : & 15.52 & 0.634 & 0.360 & 0.620 & - \\
\hline 19.09 & 0.586 & 0.350 & 0.550 & -0.220 : & 15.52 & 0.653 & 0.400 & 0.640 & - \\
\hline 18.91 & 0.586 & 0.350 & 0.550 & -0.220 : & 15.52 & 0.678 & 0.430 & 0.680 & - \\
\hline 18.78 & 0.596 & 0.365 & 0.572 & -0.140 & 15.52 & 0.736 & 0.460 & 0.720 & - \\
\hline 18.65 & 0.605 & 0.371 & 0.610 & -0.100 & 15.53 & 0.769 & - & - & - \\
\hline 18.57 & 0.639 & 0.371 & 0.626 & -0.050 & 15.50 & 0.504 & - & - & -0.055 \\
\hline 18.50 & 0.678 & 0.400 & 0.644 & -0.030 & 15.47 & 0.205 & 0.105 & 0.360 & - \\
\hline 18.42 & 0.716 & 0.411 & 0.664 & 0.000 & 15.46 & 0.243 & 0.122 & 0.370 & -0.030 \\
\hline 18.31 & 0.750 & 0.433 & 0.670 & 0.035 & 15.44 & 0.277 & 0.135 & 0.403 & -0.025 \\
\hline 18.20 & 0.774 & 0.455 & 0.687 & 0.050 & 15.40 & - & - & 0.760 & -0.080 \\
\hline 18.08 & 0.793 & 0.450 & 0.690 & 0.060 & 15.35 & - & - & - & 0.035 \\
\hline 17.94 & 0.818 & 0.451 & 0.715 & 0.070 & 15.30 & - & - & 0.820 & 0.075 \\
\hline 17.80 & 0.822 & 0.440 & 0.737 & 0.085 & 15.36 & - & - & - & 0.150 \\
\hline 17.60 & 0.822 & 0.451 & 0.735 & 0.100 & 15.40 & - & - & - & 0.185 \\
\hline 17.43 & 0.822 & 0.457 & 0.752 & 0.113 & 15.44 & - & - & - & 0.245 \\
\hline 17.24 & 0.837 & 0.465 & 0.755 & 0.135 & 15.48 & - & - & - & 0.300 \\
\hline 17.04 & 0.847 & 0.450 & 0.765 & 0.165 & & & & & \\
\hline 16.88 & 0.866 & 0.450 & 0.775 & 0.200 & & & & & \\
\hline 16.68 & 0.880 & 0.460 & 0.785 & 0.230 & & & AGB & & \\
\hline 16.34 & 0.890 & 0.495 & 0.798 & 0.270 & 15.35 & 0.706 & 0.325 & 0.770 & 0.080 \\
\hline 16.12 & 0.914 & 0.500 & 0.819 & 0.320 & 15.19 & 0.750 & 0.335 & 0.810 & 0.180 \\
\hline 15.91 & 0.938 & 0.512 & 0.837 & 0.375 & 15.04 & 0.798 & 0.343 & 0.845 & 0.280 \\
\hline 15.64 & 0.948 & 0.526 & 0.856 & 0.440 & 14.90 & 0.856 & 0.360 & 0.875 & 0.350 \\
\hline 15.43 & 0.991 & 0.540 & 0.883 & 0.480 & 14.76 & 0.899 & 0.375 & 0.915 & 0.390 \\
\hline 15.12 & 1.020 & 0.550 & 0.915 & 0.570 & 14.64 & 0.943 & 0.397 & 0.945 & 0.430 \\
\hline 14.87 & 1.069 & 0.562 & 0.946 & 0.660 & 14.53 & 0.972 & 0.420 & 0.970 & 0.450 \\
\hline 14.70 & 1.097 & 0.575 & 0.956 & 0.730 & 14.39 & 1.015 & 0.425 & 1.010 & 0.470 \\
\hline 14.39 & 1.151 & 0.598 & 1.008 & 0.840 & 14.26 & 1.054 & 0.438 & 1.000 & 0.500 \\
\hline 14.08 & 1.194 & 0.623 & 1.044 & 0.960 & 14.09 & 1.097 & 0.440 & 1.012 & 0.560 \\
\hline 13.83 & 1.247 & 0.644 & 1.076 & 1.075 & 13.91 & 1.150 & 0.452 & 1.075 & 0.680 \\
\hline 13.53 & 1.344 & 0.695 & 1.174 & 1.250 & 13.77 & 1.194 & 0.462 & 1.125 & 0.750 \\
\hline 13.32 & 1.421 & 0.735 & 1.250 & 1.460 & 13.66 & 1.223 & 0.467 & 1.165 & - \\
\hline 13.19 & 1.493 & 0.770 & 1.308 & 1.620 & 13.50 & 1.300 & - & 1.230 & - \\
\hline 13.09 & 1.546 & 0.790 & 1.360 & 1.740 & 13.38 & 1.358 & - & 1.275 & - \\
\hline 12.95 & 1.628 & 0.842 & 1.435 & 1.850 & 13.26 & 1.411 & - & 1.318 & - \\
\hline 12.95 & 1.624 & - & - & - & 13.18 & 1.464 & - & 1.400 & - \\
\hline
\end{tabular}

and can be readily used for estimates of globular-cluster $E(B-V)$ color excesses, since

$$
\begin{aligned}
E(B-V) & =(B-V)_{1}-(B-V)_{0,1} \\
& =(B-V)_{1}-0.154+1.006 \Delta_{b-1} .
\end{aligned}
$$

From our data for NGC 6723, $(B-V)_{1}=0.69 \pm 0.005$, and $\Delta_{b-1}=-0.43 \pm 0.012$. Thus, we obtain $E(B-V)=$
$0.103 \pm 0.0=15$, in excellent agreement with the values found in Sects. 4.1 and 4.2. In subsequent analysis, we adopt $E(B-V)=0.11 \pm 0.01$.

The RGB color index at the level of the horizontal branch is $(B-V)_{\mathrm{g}}=0.97$; thus, for the adopted reddening, $(B-V)_{0, \mathrm{~g}}=0.86$, and the standard color index $(B-V)_{1.4}$ becomes $(B-V)=1.51$. The RGB magnitude at the 
Table 3. Metallicity $([\mathrm{Fe} / \mathrm{H}])$ of NGC 6723 from RGB parameters

\begin{tabular}{lll}
\hline Calibration & Reference & {$[\mathrm{Fe} / \mathrm{H}]$} \\
$4.30(B-V)_{0, \mathrm{~g}}-5.00$ & Zinn \& West (1984) & -1.30 \\
$3.84(B-V)_{0, \mathrm{~g}}-4.63$ & Gratton (1987) & -1.33 \\
$4.68(B-V)_{0, \mathrm{~g}}-5.19$ & Costar \& Smith (1988) & -1.17 \\
$2.85(B-V)_{0, \mathrm{~g}}-3.76$ & Gratton \& Ortolani (1989) & -1.31 \\
\hline$-0.924 \Delta V)_{1.4}+0.913$ & Zinn \& West (1984) & -1.21 \\
$-1.01 \Delta V)_{1.4}+1.30$ & Costar \& Smith (1988) & -1.02 \\
$-0.65 \Delta V)_{1.4}+0.28$ & Gratton \& Ortolani (1989) & -1.22 \\
\hline
\end{tabular}

standard color index is $V_{1.4}=13.15$, and the elevation of the giant branch above the horizontal branch is $\Delta V_{1.4}=$ 2.30 .

There exist a number of calibrations of metallicity $[\mathrm{Fe} / \mathrm{H}]$ from $(B-V)_{0, \mathrm{~g}}$ and $\Delta V_{1.4}$. Recently Ferraro et al. (1996) presented a compilation of the relevant formulae. Table 3 contains the values of metallicity for NGC 6723 , estimated using these formulae. Almost all these values are considerably lower than the cluster's metallicity adopted earlier. The mean of these determinations is $[\mathrm{Fe} / \mathrm{H}]=$ -1.22 , rather close to the value $[\mathrm{Fe} / \mathrm{H}]=-1.26 \pm 0.09$, recently found by Fullton \& Carney (1996) from highdispersion spectra of three red giants.

According to a recent detailed study (Sandquist et al. 1996), the RR Lyraes in the globular cluster M 5 are at the magnitude $V_{\mathrm{HB}, \mathrm{M}}=15.11$, and the apparent distance modulus of M 5 is $(m-M)_{V, \mathrm{M} 5}=14.41$. Similarly, according to Buonanno et al. (1994) and Ferraro et al. (1996), for $\mathrm{M} 3 V_{\mathrm{HB}, \mathrm{M} 3}=15.67$, and $(m-M)_{V, \mathrm{M} 3}=14.94$. This leads to the absolute magnitude for RR Lyrae variables, $M_{V}(R R)=0.70$ or $M_{V}(R R)=0.73$, respectively. Different metallicities of the clusters do not significantly influence this absolute magnitude. Following Harris (1996), we adopt the slope $\partial M_{V}(R R) / \partial[\mathrm{Fe} / \mathrm{H}] \approx 0.2$. This means that, for a metallicity difference $\Delta[\mathrm{Fe} / \mathrm{H}]=0.20$, the error in the distance modulus would be only 0.04 . Adopting $M_{V}(R R)=0.72$, we obtain the apparent distance modulus $(m-M)_{V, 6723}=14.76$ for NGC 6723 . This value does not differ significantly from that quoted by Harris (1996), namely $(m-M)_{V, 6723}=14.82$.

The main-sequence turnoff is at $V_{\mathrm{TO}}=18.95 \pm$ $0.05,(B-V)_{\mathrm{TO}}=0.585 \pm 0.005$. The turnoff and the horizontal branch are separated by $\Delta V_{\mathrm{TO}}^{\mathrm{HB}}=3.50$; the same value can be obtained from CMDs using different color indices along the abscissa, the corresponding turnoff colors can be easily retrieved from Table 2 .

\subsection{The $U, U-B$ diagram}

Figure 8 shows the $(U, U-B)$ diagram for NGC 6723 and illustrates clearly that the $U$ band is especially helpful for separating the stars of bright sequences that are

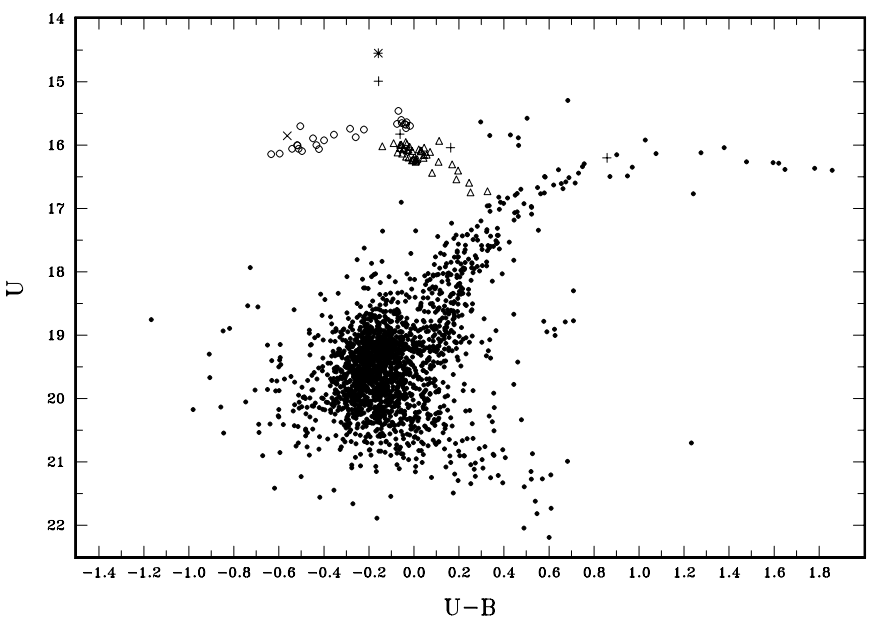

Fig. 8. The $(U, U-B)$ diagram for NGC 6723. Open circles, blue horizontal branch stars; open triangles, red horizontal branch stars; asterisk, a $U V$-bright star; plus signs $(+)$, known RR Lyraes; cross $(\times)$, new suspected variable, see text

practically overlapping in other diagrams. Note that the asymptotic giant branch is now well separated from the RGB and that the separation of red horizontal branch stars from the RGB has become clear.

The UV-bright star above the horizontal branch (asterisk) is of special interest; it was not clearly isolated in the $(V, B-V)$ diagram. Actually, the star is the standard $\mathrm{H}$ (see Table 1 ); its equatorial coordinates are $18^{\mathrm{h}} 59^{\mathrm{m}} 43^{\mathrm{s}} .8$, $-36^{\circ} 36^{\prime} 08^{\prime \prime}$ (2000.0). Its cluster membership needs confirmation.

Of special interest is the relative position of blue (open circles) and red (open triangles) horizontal branch stars. They overlap in $(U-B)$ color and show a distinctly different $U$-magnitude level near the instability strip.

A star plotted as a cross $(\times)$ sign in Figs. 6,8 and 9 appears a red horizontal branch star in Figs. 6 and 9 but as a blue horizontal branch star in Fig. 8. It might be an unknown RR Lyrae variable star, its behavior resembles that shown by known variables (plus signs). Its position, in the system of the catalog of variable stars in globular clusters (Sawyer Hogg 1973), is $x=263^{\prime \prime}, y=17^{\prime \prime}$.

\subsection{The $V, V-I$ diagram. Determination of $E_{V-I}$ and $[\mathrm{Fe} / \mathrm{H}]$ by Sarajedini's method}

In Fig. 9, we show the $(V, V-I)$ diagram for NGC 6723. From this diagram, we can determine the parameters $(V-I)_{0, \mathrm{~g}}$ and $\Delta V_{1.2}$, to be applied in the method suggested by Sarajedini (1994) for simultaneous determination of metallicity $[\mathrm{Fe} / \mathrm{H}]$ and reddening $E(V-I)$.

From our diagram, we find $(V-I)_{\mathrm{g}}=1.08 \pm 0.01$. Sarajedini's method gives $E_{V-I}=0.12 \pm 0.01$, corresponding to $E_{B-V}=0.09 \pm 0.01$, and $[\mathrm{Fe} / \mathrm{H}]=-1.77 \pm 0.10$. This reddening agrees, within errors, with other determinations, but the $[\mathrm{Fe} / \mathrm{H}]$ value is significantly lower than 


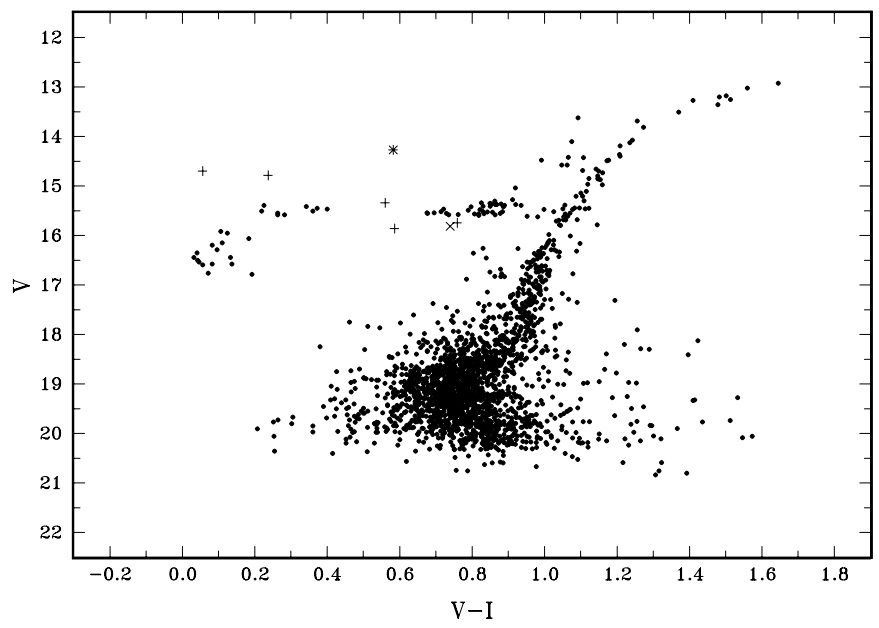

Fig. 9. The $(V, V-I)$ diagram for NGC 6723. Same symbols as in Fig. 6

all previous determinations, including those reported in Sect. 5.1. Such a low metallicity value must be incorrect owing to the method's uncertainties. However, this result continues the trend of the recent studies to lower metallicity of NGC 6723 . Indeed, the $[\mathrm{Fe} / \mathrm{H}]$ value for the cluster must be lower than it was previously believed: as noted in Sect. 4.2, the RGBs of M 3 and NGC 6723 exhibit very similar shapes in two-color diagrams as well as in the $(V, B-V)$ diagram. We note that the metallicity for $\mathrm{M} 3$ is -1.40 or lower.

\section{Age estimates}

For reasons discussed by us in detail elsewhere (see, for instance, Alcaino et al. 1998), we continue our homogeneous approach to the determination of age of globular clusters, awaiting the results of the current polemic on the controversy of globular cluster ages and the Hubble constant, in particular, with relation to the recent HIPPARCOS results. This controversy has not been completely solved until now. Chaboyer et al. (1998) argue that, from several methods including HIPPARCOS results, a consistent revision of RR Lyrae absolute magnitudes follows leading to mean age of $11.5 \pm 1.3 \mathrm{Gyr}$ for oldest globular clusters. However, Layden (1998) claims that no significant revision of the distance scale for RR Lyraes follows from HIPPARCOS data, and Frolov \& Samus (1998) obtain the distance scale for globular clusters, quite close to the traditional one, from infrared absolute magnitudes of RR Lyrae variables; these results tend to support "old" globular clusters. Fernley et al. (1998), on the base of HIPPARCOS parallaxes and proper motions, derive statistical parallaxes of RR Lyraes and obtain, for the mean age of Galactic globular clusters, the value 17.4 \pm 3.0 Gyr.

In age determinations, we use the approach similar to that used by us for M 79 (Kravtsov et al. 1997), NGC 6397
(Alcaino et al. 1997), and M 30 (Alcaino et al. 1998): we do not fix any values of reddening and distance modulus beforehand, but, vary these parameters in order to determine which isochrone best fits the observations, especially in the region of the lower giant branch and the turnoff point. The optimal position of the isochrone is determined by the best reproduction of the shape of the subgiant branch and the slope of the main sequence as well as of the color and luminosity of the turnoff. We do not attribute much physical significance to the resulting reddening values and we are going to study the reasons for these values being different from those derived by more direct methods in our future research.

Figures 10a,b,c show the $V, B-V$ diagram with ridge sequences of NGC 6723 (cf. Sect. 5.1 and Table 2), with superimposed oxygen-enhanced isochrones (Bergbusch \& VandenBerg 1992) for three $[\mathrm{Fe} / \mathrm{H}]$ values bracketing the metallicity range discussed for NGC 6723. We used the following approach to fit the ridge sequences. The HB absolute magnitude, $M_{V}(\mathrm{HB})$, is given as a function of metallicity by the relation from Harris (1996):

$M_{V}(\mathrm{HB})=0.2[\mathrm{Fe} / \mathrm{H}]+1.0$.

The apparent distance modulus is defined as $V-M_{V}=$ $V_{\mathrm{HB}}-M_{V}(\mathrm{HB})$, where for $V_{\mathrm{HB}}$ we adopted, in accordance with Sect. 5.1, the value 15.48 . The color excess $E_{B-V}$ optimal for the given chemical abundance was found from the best coincidence between the ridge sequences and the set of isochrones.

We estimated the age from the behavior of the ridge sequences in the region of the main-sequence turnoff and the subgiant branch, paying close attention to the quality of the fit of the observed sequences with the isochrones in this region for each adopted abundance.

In Fig. 10a, we present the isochrones for $[\mathrm{Fe} / \mathrm{H}]=$ -0.78 . For the chosen parameters, the agreement with the ridge sequences is excellent at the main sequence, but the observed slope of the subgiant branch is somewhat steeper than the theoretical one, and we are unable to find a unique age value for the cluster. The position of the giant branch disagrees with the cluster age that follows from the turnoff position. The resulting color excess is somewhat lower than that determined in Sect. 5.1, and the distance modulus is lower than both our value (Sect. 5.1) and the value cataloged by Harris (1996). If, however, the metallicity of NGC 6723 is really close to $[\mathrm{Fe} / \mathrm{H}]=-0.78$, then its turnoff age, in the Bergbusch \& VandenBerg scale, is $16-17$ Gyr.

Figure 10b shows isochrones for $[\mathrm{Fe} / \mathrm{H}]=-1.03$. Like in the previous case, the representation of the ridge main sequence for the adopted parameters is excellent. However, the observed slope of the subgiant branch now is close to the theoretical one, and we can also use this branch for age estimates. Agreement with theory is also found for the observed distance between the turnoff and the base of the giant branch. The observed giant branch runs along the 

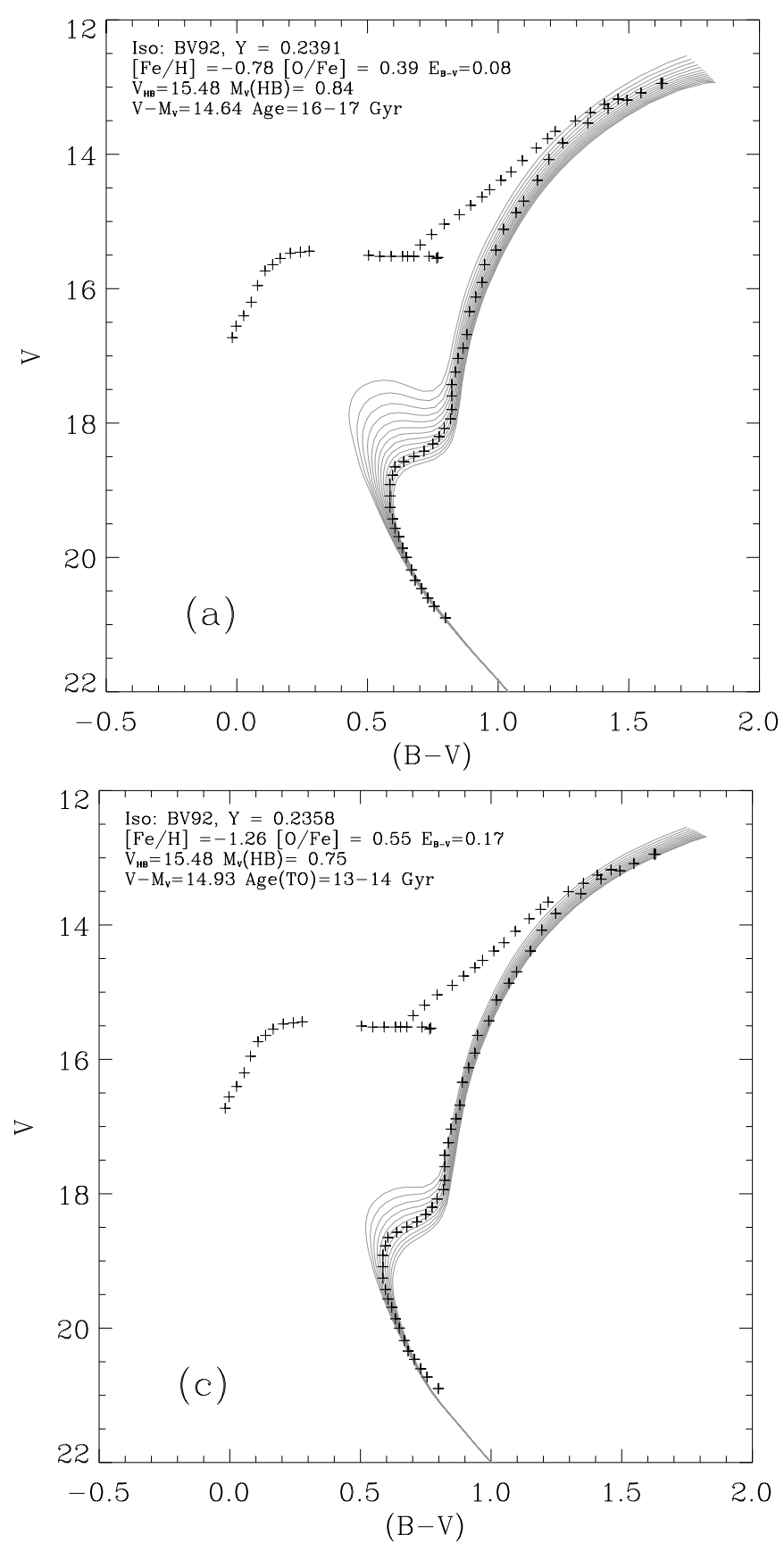

red edge of the theoretical RGBs. For $V \approx 15$ and $V \approx 13$, it is even below this edge. Note however that our red giant ridge sequence is not quite reliable for $V<15$ because of the low number of measured red giants. Still, as a whole, Fig. 10b demonstrates a much better agreement between theory and observations than Fig. 10a and Fig. 10c (described below). The color excess shows good agreement with our determination (Sect. 5.1). The distance modulus also shows better agreement, both with our determination and with the value from Harris (1996), than in the cases of Figs. 10a and 10c. If the metallicity of NGC 6723 is close to $[\mathrm{Fe} / \mathrm{H}]=-1.03$, then its age in the Bergbusch

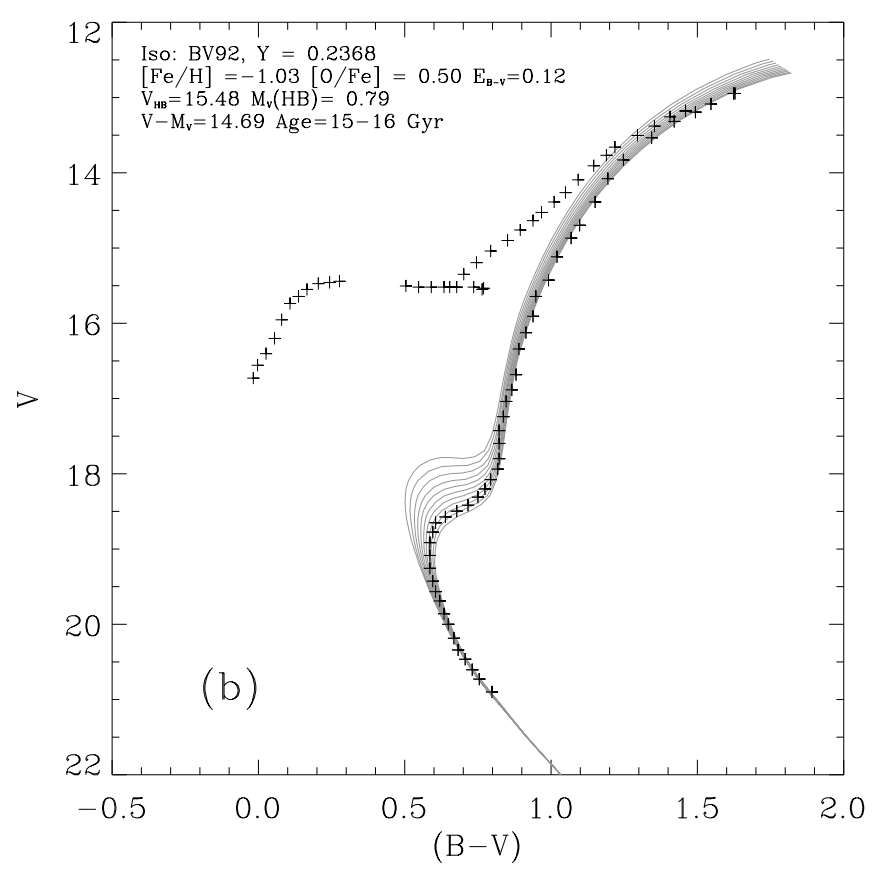

Fig. 10. The $(V, B-V)$ diagram with ridge sequences of NGC 6723 (Table 2), with superimposed oxygen enhanced isochrones of Bergbusch \& VandenBerg, for the indicated adopted parameters. a) $[\mathrm{Fe} / \mathrm{H}]=-0.78 ; \mathbf{b})[\mathrm{Fe} / \mathrm{H}]=-1.03 ; \mathbf{c})$ $[\mathrm{Fe} / \mathrm{H}]=-1.26$. The $M_{V}(\mathrm{HB})$ values indicated in each panel are from Eq. (1), for the given metallicity value; for panel c) this value does not agree with the adopted distance modulus, as discussed in the text. The isochrones span the age range from 5 to 18 Gyr (panel a) and from 8 to 18 Gyr (panels b and $\mathbf{c}$ ), the increment in age between subsequent isochrones is $1 \mathrm{Gyr}$

\& VandenBerg scale, from the turnoff and the subgiant branch, is $15-16$ Gyr.

Figure 10c presents Bergbusch \& VandenBerg isochrones for $[\mathrm{Fe} / \mathrm{H}]=-1.26$, the metallicity value closest to the modern estimates for NGC 6723. For the adopted parameters, the agreement with the ridge sequences for the main sequence is the worst of the three cases. The observed slope of the subgiant branch agrees with the theoretical one. The observed lower giant branch tends towards the blue edge of the theoretical RGBs, i.e. towards lowest ages in the Bergbusch \& VandenBerg set of isochrones, and its position does not agree with turnoff ages. The color excess is significantly higher than our 
determination (Sect. 5.1), and the distance modulus exceeds both our value and the value quoted by Harris (1996). The agreement between the theoretical and the observed diagrams is achieved at $V-M_{V}=V_{\mathrm{HB}}-M_{V}(\mathrm{HB})+$ 0.2 . If, however, the metallicity of NGC 6723 is really close to $[\mathrm{Fe} / \mathrm{H}]=-1.26$, its age in the Bergbusch \& VandenBerg scale, from the turnoff and the subgiant branch, would be 13 - 14 Gyr, the minimum value among the discussed cases.

We conclude that, from the point of view of the agreement between the theoretical models and the observed sequences, the best metallicity value is close to $[\mathrm{Fe} / \mathrm{H}]=$ -1.03 . It leads to the cluster's photometric parameters (reddening and distance modulus) being in satisfactory agreement with independent estimates and to the age of $15-16$ Gyr in the scale of Bergbusch \& VandenBerg.

\section{Conclusions}

From our CCD photometry of 3620 stars in the globular cluster NGC 6723, we have determined several important parameters for this cluster. Its color excess is $E(B-V)=0.11 \pm 0.01$. The most plausible value of metallicity is $[\mathrm{Fe} / \mathrm{H}]=-1.22 \pm 0.15$; even lower values are possible. Note that the method suggested by Sarajedini (1994) leads to an unacceptably low metallicity for NGC 6723. The apparent distance modulus of the cluster is $(m-M)_{V, 6723}=14.76$. From isochrone analysis, we prefer the NGC 6752 age in the range between 15 and $16 \mathrm{Gyr}$, in the scale of Bergbusch \& VandenBerg (1992). We have found an interesting separation of blue and red horizontal branches, near the region of the instability strip, by $U$ magnitude in the $(U, U-B)$ diagram.

The shape and slope of the RGB in NGC 6723 which we derive are practically the same as in M 3. Remarkably, our value $\Delta V_{\mathrm{TO}}^{\mathrm{HB}}=3.50$ for NGC 6723 is almost exactly the same as those found by other authors for M 3 (3.52; Ferraro et al. 1996) and for M 5 (3.47; Sandquist et al. 1996). This similarity of the three clusters with intermediate morphology of the horizontal branch should be taken into account when analyzing the "second parameter" problem.

Acknowledgements. We wish to thank Mr. Oleg Lahtionov for assistance during this study.

\section{References}

Alcaino G., Liller W., Alvarado F., et al., 1997, AJ 114, 1067 Alcaino G., Liller W., Alvarado F., et al., 1998, AJ 115, 1492
Alvarado F., Wenderoth E., Alcaino G., Liller W., 1994, AJ 107,631

Bergbusch P.A., VandenBerg D.A., 1992, ApJS 81, 163

Buonanno R., Corsi C.E., Buzzoni A., et al., 1994, A\&A 290, 69

Buser R., Kurucz R.L., 1992, A\&A 264, 557

Butler D., 1975, ApJ 200, 68

Chaboyer B., Demarque P., Kernan P.J., Krauss L.M., 1998, ApJ 494, 96

Costar D., Smith H.A., 1988, AJ 96, 1925

Fernley J., Barnes T.G., Skillen I., et al., 1998, A\&A 330, 515

Ferraro F.R., Carretta E., Corsi C.E., et al., 1996, A\&A 320, 757

Frolov M.S., Samus N.N., 1998, Astron. Lett. 24, 171

Fullton L.K., 1996, PASP 108, 545

Fullton L.K., Carney B.W., 1993, BAAS 25, 1407

Fullton L.K., Carney B.W., 1996, in: Formation of the Galactic Halo ... Inside and Out, PASPC, 92, Morrison M. and Sarajedini A. (eds.), p. 265

Gascoine S.C.B., Ogston F.A., 1963, Observatory 83, 64

Gratton R.G., 1987, A\&A 179, 181

Gratton R.G., Ortolani S., 1989, A\&A 211, 41

Grebel E.K., Roberts W.J., 1995, A\&AS 109, 293

Harris W.E., 1996, AJ 112, 1487

Kinman T.D., 1959, MNRAS 119, 538

Kravtsov V., Ipatov A., Samus N., et al., 1997, A\&AS 125, 1

Kron G.E., Mayall N.U., 1960, AJ 65, 581

Kurucz R.L., 1993, Model Atmospheres CD-ROMs. Smithsonian Astrophysical Observatory

Layden A.C., 1998, in: Post-Hipparcos Cosmic Candles, Heck A. and Caputo F. (eds.). Dordrecht: Kluwer (in press)

Lee Y.-W., Demarque P., Zinn R., 1988, in: Calibration of Stellar Ages, A.G. Davis Philip (ed.). Schenectady: L. Davis Press, p. 149

Lee Y.-W., 1992, AJ 104, 1780

Martins D.H., Fraquelli D.A., 1987, ApJS 65, 83

Menzies J., 1974, MNRAS 168, 177

Mironov A.V., 1972, SvA 16, 105

Mironov A.V., Samus N.N., 1974, Peremennye Zvyozdy 19, 337 (in Russian)

Rosino L., 1965, Kleine Veröff. Remeis-Sternw. Bamberg, IV, No. 40,98

Samus N., Ipatov A., Smirnov O., et al., 1995a, A\&AS 112, 439

Sandquist E.L., Bolte M., Stetson P.B., Hesser J.E., 1996, ApJ 470,910

Sarajedini A., 1994, AJ 107, 618

Sawyer Hogg H., 1973, Publ. David Dunlap Obs. 3, No. 6

Smith H.A., 1981, ApJ 250, 719

Smith G.H., Hesser J.E., 1986, PASP 98, 838

Stetson P.B., 1991a, DAOPHOT II Users Manual. Victoria: Dominion Astrophysical Observatory

Zinn R., West M.J., 1984, ApJS 55, 45 